\title{
INTERCELL INTERFERENCE CANCELLATION AT A WCDMA MOBILE TERMINAL BY EXPLOITING EXCESS CODES
}

\author{
Massimiliano Lenardi, Abdelkader Medles and Dirk T. M. Slock \\ Mobile Communications Department - Institut Eurécom * \\ 2229 Route des Crêtes, B.P. 193, F-06904 Sophia Antipolis Cedex, FRANCE \\ E-mail: $\{l e n a r d i$, medles, slock\}@eurecom. fr
}

\begin{abstract}
The deployment of third generation CDMAbased wireless systems foresees a loading fraction that is smaller than one, i.e. the number of users per cell is scheduled to be significantly less than the spreading factor to attain an acceptable performance. This means that a base station canset apart a subset of the codes, the excess codes, that it will not use. The existence of excess codes implies the existence of a noise subspace, which can be used to cancel the interference coming from a neighboring base station. In the case of aperiodic codes (such as in the FDD mode of UMTS), the noise subspace is time-varying due to the scrambling. This motivated us to introduce structured receivers that combine scrambling and descrambling operations with projections on code subspaces and linear time-invariant filters for equalization, interference cancellation and multipath combining. So the time-varying part of these receivers is limited to (de-)scrambling operations. The design of the various filter parts gets discussed. Performance improvements are illustrated via simulations.
\end{abstract}

\section{DOWNLINK SIGNAL MODEL}

Fig. 1 shows the downlink signal model in baseband. For each base station $j, j=1,2$, the $K^{j}$ users are assumed to transmit linearly modulated signals over the same linear multipath channel $h^{j}(t)$; the BS 2 is considered the interfering one. Additive noise $v(t)$ is then included in the received signal $y(t)$.

Without loss of generality, we consider here the case of one interfering base station. The symbol and chip periods $T$ and $T_{c}$ are related through the spreading factor $L: T=L T_{c}$, which is assumed here to be common for all the users and for the two base stations. The total chip sequences $b_{l}^{j}$ are the sum of the chip sequences of all the users for each BS $j$. Every user chip sequence is given by the product between the $n$th symbol of the $k$ th user and an aperiodic spreading sequence $w_{k, l}^{j}$ which is itself the product of a periodic Walsh-Hadamard (with unit energy) spreading sequence $\boldsymbol{c}_{k}^{j}=\left[\begin{array}{llll}\boldsymbol{c}_{k, 0}^{j} & \boldsymbol{c}_{k, 1}^{j} & \cdots & \boldsymbol{c}_{k, L-1}^{j}\end{array}\right]^{T}$, and a basestation specific unit magnitude complex scrambling sequence $s_{l}^{j}$ with variance $1, w_{k, l}^{j}=c_{k, l \bmod L}^{j} s_{l}^{j}$ :

$$
b_{l}^{j}=\sum_{k=1}^{K^{j}} b_{k, l}^{j}=\sum_{k=1}^{K^{j}} a_{k,\left\lfloor\frac{l}{L}\right\rfloor}^{j} w_{k, l}^{j} \quad j=1,2 .
$$

The scrambling operation is a multiplication of chip rate sequences. The spreading operation is represented by a filtering of an upsampled symbol sequence with the spreading sequence as

\footnotetext{
*Eurécom's research is partially supported by its industrial partners: Ascom, Swisscom, France Télécom, La fondation CEGETEL, Bouygues Télécom, Thales, ST Microelectronics, Motorola, Hitachi Europe and Texas Instruments. The work leading to this paper was also partially supported by the French RNRT (National Network for Telecommunications Research) project AUBE.
}

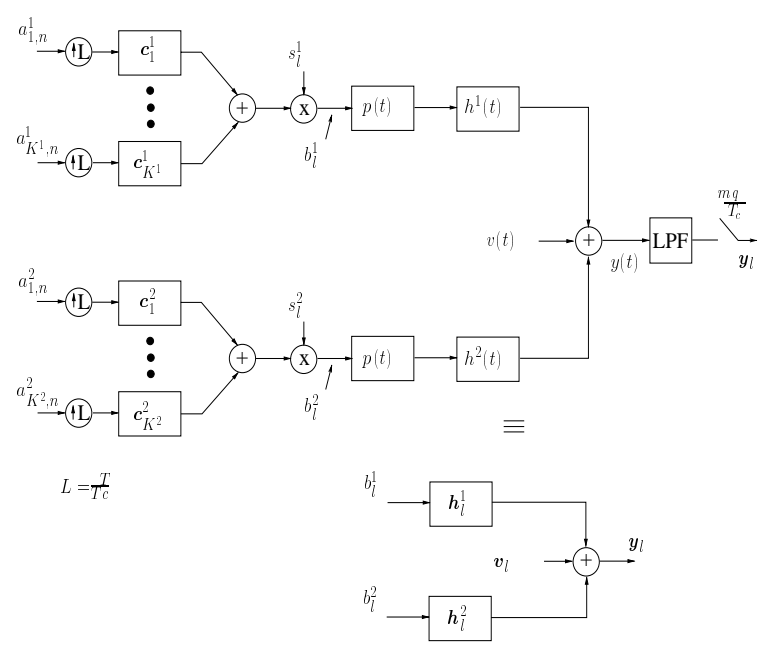

Figure 1. Downlink signal model

impulse response. The chip sequences $b_{l}^{1,2}$ get transformed into a continuous-time signals by filtering them with the pulse shape $p(t)$ and then passing them through the multipath propagation channels $h^{1}(t)$ and $h^{2}(t)(q \times 1)$ (from BS 1 and from BS 2 to the $q$ sensors of the mobile station respectively) to yield the total received signal $y(t)$. The receiver samples $m$ times per chip period the lowpass filtered received signal. Taking into account the use of the $q$ receive antennas also, the total number of samples per chip period becomes $m q$. Stacking the $m q$ samples per chip period in vectors, we get for the sampled received signal

$$
\boldsymbol{y}_{l}=\boldsymbol{y}_{l}^{1}+\boldsymbol{y}_{l}^{2}+\boldsymbol{v}_{l}, \quad \boldsymbol{y}_{l}^{j}=\sum_{k=1}^{K^{j}} \sum_{i=0}^{N-1} \boldsymbol{h}_{i}^{j} b_{k, l-i}^{j} \quad j=1,2
$$

where

$$
\boldsymbol{y}_{l}^{j}=\left[\begin{array}{c}
y_{1, l}^{j} \\
\vdots \\
y_{m q, l}^{j}
\end{array}\right], \boldsymbol{h}_{l}^{j}=\left[\begin{array}{c}
h_{1, l}^{j} \\
\vdots \\
h_{m q, l}^{j}
\end{array}\right], \boldsymbol{v}_{l}=\left[\begin{array}{c}
v_{1, l} \\
\vdots \\
v_{m q, l}
\end{array}\right]
$$

Here $h_{l}^{j}(m q \times 1)$ represents the vectorized samples (represented at chip rate) of the overall channel $h^{j}(t)$, including pulse shape, propagation channel and receiver filter. The overall channels $h^{j}(t)$ are assumed to have the same delay spread of $N$ chips.

In the case in which the scrambling sequence is aperiodic and in which we model it as also the symbol sequences as independent i.i.d. sequences, then the chip sequences $b_{l}^{1,2}$ are sums of independent white noises (chip rate i.i.d. sequences, hence stationary) and hence are white noises. The intracell contribution to $\boldsymbol{y}_{l}$ then is a stationary (vector) process (the continuous-time counterpart is cyclostationary with chip period). The intercell 
interference is a sum of contributions that are of the same form as the intracell contribution so its contribution to $\boldsymbol{y}_{l}$ is also a stationary vector process. The remaining noise is assumed to be white stationary noise. Hence the sum of intercell interference and noise, $\boldsymbol{v}_{l}$, represented at chip rate, is stationary.

\section{RECEIVER STRUCTURES FOR INTERCELL IN- TERFERENCE CANCELLATION}

In this section we focus on the description of the receiver structures that we analyzed and implemented.

\section{II.1. Linear IC Structures}

Fig. 2 shows a linear receiver in which a descrambler and a correlator follow a general FIR filter $f_{l}$ whose input is at sampling rate and its output is at chip rate. In the case of a RAKE

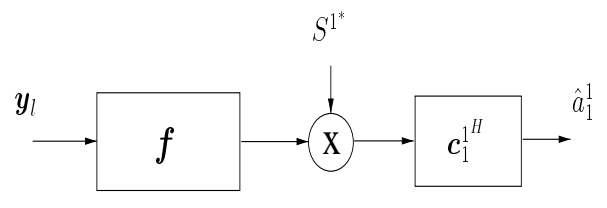

Figure 2. Filter-Correlator Receiver Structure.

receiver implementation, $f$ is the channel matched filter $h^{1^{H}}$ which is equivalent to considering the BS 2 signal as an additive noise and to maximize the SNR at the filter output. To maximize the SINR at the receiver output while taking into account the intracell interference, the filter $f$ can be adapted to be the Max-SINR filter (see [1], [2]). The RAKE receiver is a reference structure. The Max SINR receiver takes (intra and intercell) interference into account by modeling it as chip rate cyclostationary noise. The other structures to be considered below take more structural information about the interference into account.

\section{II.2. Hybrid IC Structures}

Fig. 3 shows one instance of a hybrid structure in which an Interference Cancellation (IC) Branch preceeds a linear receiver as in Fig. 2. The IC branch is formed by an equalizer, a descrambler, a projector, a scrambler and a filter; different implementation are possible and are presented in the following subsections 1 to 3. This approach is conceived to perform intracell or intercell interference cancellation in order to improve the performance of the linear receivers presented above by exploiting the structure of the received signal itself (the IC branch is indeed equivalent to a time-variant filter).

\section{IC Structure 1}

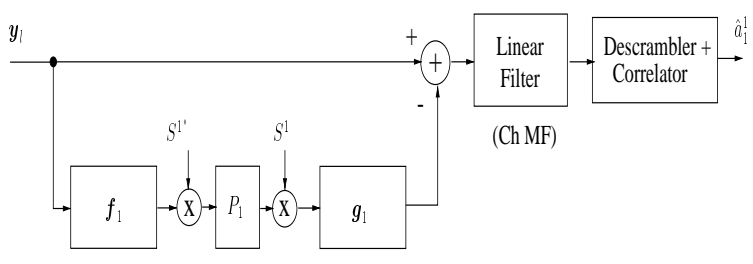

Figure 3. Hybrid Structure 1, intracell IC.

Here the base station 1 is considered in the IC branch and intracell interference cancellation is performed by exploiting the orthogonality between user codes of BS 1 after the equalizer and projecting this signal into the subspace of the used spreading codes, except for the user of interest. Then, by "re-channeling" the rescrambled output, the intracell interference can be subtructed from the received signal $\boldsymbol{y}_{l}$ and a RAKE receiver can be applied to extract the signal of interest. This structure allows also to decrease the intercell interference plus noise somewhat due to the projection operation. The equalizer could be either a Zero-Forcing (ZF) or a Minimun Mean Square Error (MMSE) equalizer: the former enhances more the intercell interference plus noise but preserves the code orthogonality, while the latter perturbs the structure of the signal received from the BS 1 but does not increase so much the intercell interference plus noise (see [3]). The "re-channeling" filter could be either the channel $h^{1}$ or a Wiener (LMMSE) filter.

\section{IC Structure 2}

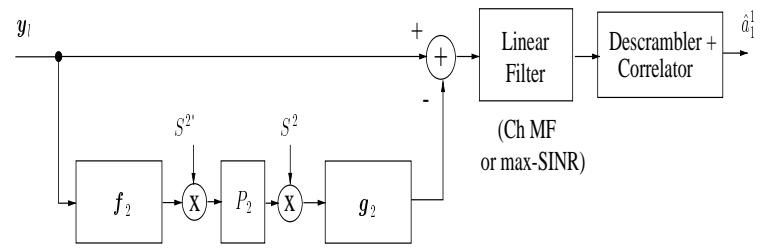

Figure 4. Hybrid Structure 2, intercell IC

In this structure, the BS 2 is considered in the IC branch; the equalizer and the "re-channeling" filter can again be described as in section 1 , but the projection is onto the complete subspace of used spreading codes of BS 2. In this approach it is possible to implement either a RAKE receiver or a max-SINR receiver after the subtractor, the latter being conceivable due to the absence of signal from BS 2 in the IC error signal.

\section{IC Structure 3}

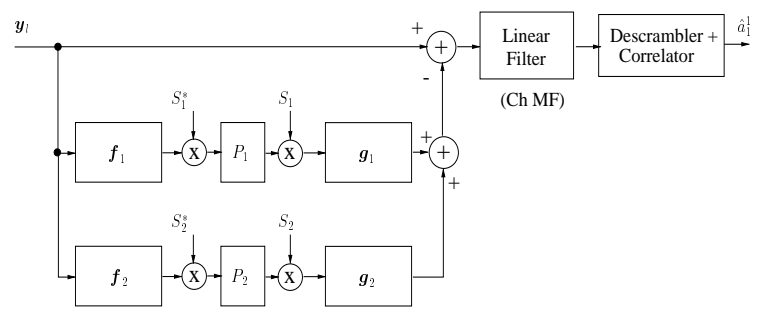

Figure 5. Hybrid Structure with two IC branches

This structure combines the single-branch approaches of sections 1 and 2. The equalizers and the projections are defined as before, while the two filters $f_{1}$ and $f_{2}$ can be equal to the corresponding BS channels $h^{1}$ and $h^{2}$ or jointly adapted to have minimum interference plus noise variance at the subtractor output. The linear receiver after the subtractor can be in this case just a RAKE receiver, due to the simultaneous cancellation of intracell and intercell interference.

\section{POLYNOMIAL EXPANSION (PE) IC STRUCTURES}

In this section we develop new intra and intercell interference cancelling structures based on polynomial expansion (see [4]).

\section{III.1. Symbol Rate PE IC Structure}

A vector of received signal over one symbol period $n$ can be written as:

$\boldsymbol{Y}[n]=\boldsymbol{H}_{1}(z) \boldsymbol{S}_{1}[n] \boldsymbol{C}_{1} \boldsymbol{A}_{1}[n]+\boldsymbol{H}_{2}(z) \boldsymbol{S}_{2}[n] \boldsymbol{C}_{2} \boldsymbol{A}_{2}[n]+\boldsymbol{V}[n]$ 
As shown in Fig. $6, \boldsymbol{H}_{j}(z)=\sum_{i=0}^{M_{j}-1} \boldsymbol{H}[i] z^{-i}$ is the $L m q \times L$ channel transfer function at symbol rate. The block coeffcients $\boldsymbol{H}_{j}[i]$ correspond to the $M_{j}=\left\lceil\frac{L+N_{j}+d_{j}-1}{L}\right\rceil$ parts of the block Toeplitz matrix (with $m q \times 1$ sized blocks) with $h^{j}$ as first column. $d_{j}$ is the TX delay between BS $j$ and the mobile, expressed in chip periods. $h^{j}$ comprises the delay in the channel and hence its top entries may be zero. The $L \times L$ matrices $\boldsymbol{S}_{j}[n]$ are diagonal and contain the scrambler of BS $j$ for symbol period $n$. The

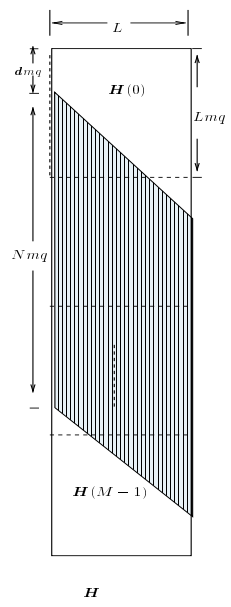

Figure 6. Channel impulse response $\boldsymbol{H}(z)$.

vectors $\boldsymbol{A}_{j}$ contain the $K_{j}$ symbols of BS $j$ for symbol period $n$, and $C_{j}$ is the $L \times K_{j}$ matrix of the $K_{j}$ active codes for BS $j$. Equation (4) can be rewritten as

$$
\begin{aligned}
& \boldsymbol{Y}[n]=\left[\boldsymbol{H}_{1}(z) \boldsymbol{S}_{1}[n] \boldsymbol{C}_{1} \quad \boldsymbol{H}_{2}(z) \boldsymbol{S}_{2}[n] \boldsymbol{C}_{2}\right] \boldsymbol{A}[n]+\boldsymbol{V}[n](5) \\
& =\widetilde{G}(z) A[n]+V[n]
\end{aligned}
$$

where $\boldsymbol{A}$ is the $\left(K=K_{1}+K_{2}\right) \times 1$ vector containing all the transmitted symbols for the two BS. $\widetilde{G}(z)$ is a $L m q \times K$ channel-plus-spreading symbol rate filter, and is time-varying due to the scrambling. The condition $K<L q$ (or even $K<L m q$ ) guarantees the existense of an FIR left inverse filter for $\widetilde{\boldsymbol{G}}(z)$, and allows zero-forcing interference cancellation. The calculation of an exact inverse of the channel would lead to high complexity due to the time-varying nature of the scrambler. Hence we will provide a good approximation to this inverse with acceptable complexity based on polynomial expansion [4].

Let $\boldsymbol{X}[n]$ be the $K \times 1$ correlator output, then:

$$
\begin{aligned}
\boldsymbol{X}[n] & =\widetilde{\boldsymbol{F}}(z) \boldsymbol{Y}[n] \\
& =\left[\begin{array}{c}
\boldsymbol{C}_{1}^{H} \boldsymbol{S}_{1}^{H}[n] \boldsymbol{F}_{1}(z) \\
\boldsymbol{C}_{2}^{H} \boldsymbol{S}_{2}^{H}[n] \boldsymbol{F}_{2}(z)
\end{array}\right](\tilde{\boldsymbol{G}}(z) \boldsymbol{A}[n]+\boldsymbol{V}[n]) \\
& =\boldsymbol{M}(z) \boldsymbol{A}[n]+\widetilde{\boldsymbol{F}}(z) \boldsymbol{V}[n]
\end{aligned}
$$

where $\boldsymbol{F}_{j}(z)=\frac{\boldsymbol{H}_{j}^{H}\left(z^{-*}\right)}{\left\|h_{j}\right\|^{2}}$ are the channel matched filters and $\boldsymbol{M}(z)=\widetilde{\boldsymbol{F}}(z) \widetilde{\boldsymbol{G}}(z)$. Let $\boldsymbol{F}_{j}(z) \boldsymbol{H}_{j}(z)=\boldsymbol{D}_{j j}(z)=$ $\sum_{i} D_{j j}[i] z^{-i}$. We assume that $\operatorname{diag}\left\{\boldsymbol{D}_{j j}[0]\right\}=I$. As a result, if $M(z)=\sum_{i} M[i] z^{-i}, \operatorname{diag}\{M[0]\}=I$. In order to obtain the estimate of $\boldsymbol{A}[n]$, we initially consider the processing of $\boldsymbol{X}[n]$ by a decorrelator:

$$
\begin{aligned}
\widehat{A}[n] & =M(z)^{-1} \boldsymbol{X}[n] \\
& =(I+\bar{M}(z))^{-1} \boldsymbol{X}[n]
\end{aligned}
$$

The correlation matrix $M(z)$ has a coefficient $M[0]$ with a dominant unit diagonal in the sense that all other elements of the $M[i]$ are much smaller than one in magnitude. Hence, the polynomial expansion approach suggests to develop ( $I+$ $\bar{M}(z))^{-1}=\sum_{i=0}^{\infty}(-\bar{M}(z))^{i}$ up to some finite order, which leads to

$$
\begin{gathered}
\hat{\boldsymbol{A}}^{(-1)}[n]=0 \\
i \geq 0 \quad \hat{\boldsymbol{A}}^{(i)}[n]=\boldsymbol{X}[n]-\bar{M}(z) \hat{\boldsymbol{A}}^{(i-1)}[n] .
\end{gathered}
$$

In practice, we may want to stop at the first-order expansion, the quality of which depends on the degree of dominance of the diagonal of the static part of $M(z)$ with respect to its off-diagonal elements and the dynamic part.

At first order, the expression for the user of interest (user one) becomes:

$$
\begin{aligned}
\hat{\boldsymbol{a}}_{1}^{1}[n] & =\boldsymbol{e}_{1}^{H} \hat{\boldsymbol{A}}^{(1)}[n] \\
& =\boldsymbol{e}_{1}^{H}\left(\boldsymbol{X}[n]-\overline{\boldsymbol{M}}(z) \hat{\boldsymbol{A}}^{(0)}[n]\right) \\
& =\boldsymbol{e}_{1}^{H}(2 \boldsymbol{X}[n]-\boldsymbol{M}(z) X[n]) \\
& =\boldsymbol{e}_{1}^{H} \widetilde{\boldsymbol{F}}(z)(2 \boldsymbol{Y}[n]-\widetilde{\boldsymbol{G}}(z) X[n]) \\
& =\boldsymbol{c}_{1}^{1}{ }^{H} \boldsymbol{S}_{1}^{H}[n] \boldsymbol{F}_{1}(z)(2 \boldsymbol{Y}[n]-\widetilde{\boldsymbol{G}}(z) \widetilde{\boldsymbol{F}}(z) \boldsymbol{Y}[n])
\end{aligned}
$$

The corresponding structure is shown in Fig. 7, where $\boldsymbol{P}_{j}$ denotes the projection on all the active codes $C_{j}$ of $\mathrm{BS}$ j, (in the Hybrid Structures considered earlier, $\boldsymbol{P}_{1}$ denoted the projection onto the interfering active codes of BS 1).

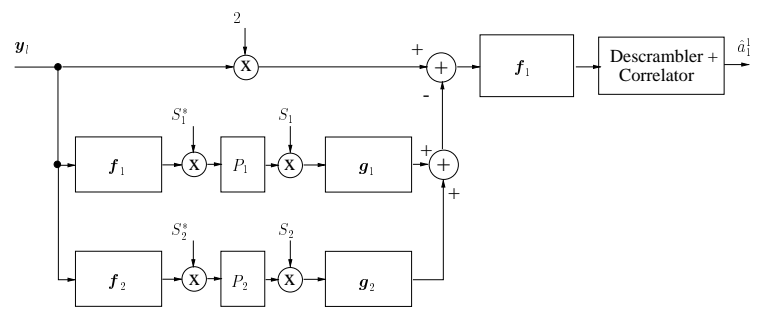

Figure 7. Symbol Rate Polynomial Expansion Structure.

In our developement so far (decorrelator approach) the equalizer $f_{1}$ (resp. $f_{2}$ ) was the normalized matched filter to $h_{1}$ (resp $h_{2}$ ). An improved approach consists of using MMSE chip rate equalizers for the respective BS channels, normalized to have $f_{1} h_{1}=1$ (resp. $f_{2} h_{2}=1$ ). The idea behind this modification is to make the diagonal more dominant, leading to a more accurate first order expansion for the inverse. A second modification consists of replacing the rechanneling filters $\boldsymbol{g}_{j}$, which were so far the channel impulse responses $h_{j}$, by Wiener filters so that the polynomial expansion moves from a decorrelator approach to a MMSE approach. It appears that taking $g_{j}$ to be a scalar multiple of $h_{j}$, in which the scalar is adjusted for MMSE after the subtraction process, may yield most of the attainable improvement.

\section{III.2. Chip Rate PE IC Structure}

In the symbol rate approach above, polynomial expansion was applied to the composite operation of spreading, scrambling, channeling, equalization, descrambling and despreading. This composite operation takes symbol rate inputs and outputs. Alternatively, we may want to apply the polynomial expansion to only the channeling and equalization operations, in which case we take chip rate inputs and outputs. To take advantage of the previously introduced notation, we shall nevertheless consider a 
representation at the symbol rate

$$
\begin{aligned}
Z[n]= & \boldsymbol{F}(z) \boldsymbol{Y}[n]=\left[\begin{array}{l}
\boldsymbol{F}_{1}(z) \\
\boldsymbol{F}_{2}(z)
\end{array}\right] \boldsymbol{Y}[n] \\
= & \boldsymbol{F}(z)(\boldsymbol{G}(z) \tilde{\boldsymbol{A}}[n]+\boldsymbol{V}[n]) \\
= & \boldsymbol{F}(z)\left(\left[\boldsymbol{G}_{1}(z) \boldsymbol{G}_{2}(z)\right]\left[\begin{array}{cc}
\boldsymbol{S}_{1}[n] \boldsymbol{C}_{1} & 0 \\
0 & \boldsymbol{S}_{2}[n] \boldsymbol{C}_{2}
\end{array}\right] \boldsymbol{A}[n]\right. \\
& +\boldsymbol{V}[n]) \\
= & \boldsymbol{D}(z) \tilde{\boldsymbol{A}}[n]+\boldsymbol{F}(z) \boldsymbol{V}[n]
\end{aligned}
$$

A good approximation of the inverse is:

$$
\begin{aligned}
D(z)^{-1} & =(I+\bar{D}(z))^{-1} \\
& \approx I-\bar{D}(z) \\
& \approx 2 I-D(z)
\end{aligned}
$$

The first order symbol estimation for user of interest is then:

$$
\begin{aligned}
\hat{\boldsymbol{a}}_{1}^{1}[n] & =\left[\begin{array}{ll}
\boldsymbol{c}_{1}^{1 H} \boldsymbol{S}_{1}^{H}[n] & 0
\end{array}\right](2 \boldsymbol{I}-\boldsymbol{D}(z)) \boldsymbol{Z}[n] \\
& =\left[\begin{array}{ll}
\boldsymbol{c}_{1}^{1 H} \boldsymbol{S}_{1}^{H}[n] & 0
\end{array}\right](2 \boldsymbol{I}-\boldsymbol{F}(z) \boldsymbol{G}(z)) \boldsymbol{Z}[n] \\
& =\boldsymbol{c}_{1}^{1 H} \boldsymbol{S}_{1}^{H}[n] \boldsymbol{F}_{1}(z)(2 \boldsymbol{Y}[n]-\boldsymbol{G}(z) \boldsymbol{F}(z) \boldsymbol{Y}[n])
\end{aligned}
$$

The implementation of this receiver corresponds to Fig. 7 but without the projection and (de)scrambling operations.

\section{SOFT HANDOVER RECEIVER STRUCTURES}

In the case of soft handover, the signal of interest (assume user 1) is transmitted by (e.g.) two BS. The proper modification of the end part of the receivers, which so far consisted of a filtercorrelator part for BS 1 and user 1, becomes the sum of two such branches, as indicated in Fig. 8.

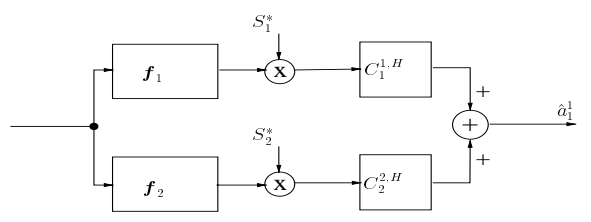

Figure 8. Soft handover receiver end.

\section{NUMERICAL EXAMPLES}

Figures 9 to 15 present some of the simulations that we have performed to evaluate the various structures. In the legends of these figures, MS refers to max-SINR receiver. The $K^{j}$ users of base station $j$ are considered synchronous between them, with the same spreading factor $L=32$ and using the same downlink channel $h^{j}$ which is a FIR filter, convolution of a sparse Vehicular A UMTS channel and a pulse shape (root-raised cosine with roll-off factor of 0.22 ). The channel length is $N=19$ chips, due to the UMTS chip rate of $3.84 \mathrm{Mchips} / \mathrm{sec}$. An oversampling factor of $m=2$ and one receive antenna $q=1$ are used. Two possible user power distributions are simulated: all interferers (intracell and intercell) have the same power and the user of interest has either the same power also or 10dB less power (nearfar situation). In Fig. 9 to Fig. 12: the first two curves in the legend are the ones described in section II.1, namely the RAKE and the max-SINR receivers; the curves three to six in the legend are referring to the structures in which the "re-channeling" filters are the channels themselves, $g_{j}=h^{j} j=1,2$, while the last four curves in the legend are the same structures but with $g_{j}$ optimized as explained in section 1 . The names in the legend "Si-x-RAKE" or "Si-x-MS" refer to hybrid structure i with "re-channeling" filter x (channel or optimized filter) and output linear filter RAKE or max-SINR. In the case of hybrid structure 3 (section 3) $\mathrm{H}$ refers to the use of the two channels $h^{1}$ and $h^{2}$ and $\mathrm{G}$ to the use of the jointly optimized filters $\boldsymbol{g}_{1}$ and $\boldsymbol{g}_{2}$.

In Fig. 9, all the curves are above the RAKE curve. Another remark is that when a MMSE equalizer is implemented, the optimization of the filters $\boldsymbol{g}_{j}$ yields little improvement over the use of channel matched filters (see [3]). So the following figures always assume the use of MMSE equalizers and in this case the hybrid structure 3 works always much better than the other hybrid structures. Fig. 10 confirms even more this result showing the case of a near-far situation for the user of interest; the hybrid structure 3 is the best one for the complete range of interest for $\mathrm{Eb} / \mathrm{No}$.

Fig. 11 and Fig. 12 show that all the structures are sensitive to the number of users in the system, but again hybrid structure 3 is better because it is less sensitive, especially when the "rechanneling" filters are optmized.

In Fig. 13 to Fig. 15, "MMSE-PE" in the legend refers to the first order symbol rate polynomial expansion structure which uses (chip rate) MMSE equalizers, and "RAKE-PE" refers to the use of matched filters as equalizers.

Fig. 13 shows that the "MMSE-PE" structure performs better than hybrid structure 3 , and hence is the best of all the structures studied, despite being less complex because it does not need optimization of the "re-channeling" filter.

Fig. 14 and Fig. 15 show that the first order (symbol rate) polynomial expansion structure (using either channel matched filters of MMSE equalizers for the $f_{j}$ ) are sensitive to the number of users in the system, but this sensitivity appears to be acceptable in the case of the "MMSE-PE" since its performance remains the best for reasonable Eb/No. We believe that inserting a scaling factor in the subtraction branch, that is optimized for MMSE of the subtraction error, should eliminate the performance degradation at low Eb/No.

\section{CONCLUSIONS}

In the case of aperiodic scrambling, the classical RAKE receiver exhibits siginificant limitations when a strong interfering base station is present. The Max-SINR receiver, which is a MMSE equalizer-correlator cascade, allows improved performance. Still better performance can be achieved when more knowledge about the signal structure gets exploited. We proposed two receiver structures that exploit the existence of excess (unused) codes. One type of structure, called hybrid structure, was developed based on intuitive ideas about noise cancellation in the direction of unused codes. The other structure is based on the more formal polynomial expansion approach. We have observed that the two approaches yield very similar structures. In the case of polynomial expansion, we also introduced a chip rate approach, in which only the scrambling sequences of the base stations are exploited (and not the excess codes). The proposed structures have a reasonable complexity and allow important performance gains. In particular, the structures exhibit no SINR saturation at high SNR (in contrast to the RAKE receiver) which means that all interference can be forced to zero.

\section{References}

[1] Y. -P.E. Wang and G. E. Bottomley, "Generalized RAKE Reception for Cancelling Interference from Multiple Base stations," in Proc. Fall VTC 2000, (Boston, Massachusset USA), September 2000. 
[2] M. Lenardi and D. T. M. Slock, "A RAKE Receiver with Intracell Interference Cancellation for a DS-CDMA Synchronous Downlink with Orthogonal Codes," in Proc. VTC 2000, (Tokyo, Japan), May 2000.

[3] A. M. Massimiliano Lenardi and D. T. M. Slock, "Downlink Intercell Interference Cancellation in WCDMA by Exploiting Excess Codes," in Proc. SAM 2000, (Boston, Massachusset - USA), March 2000.

[4] S. Moshavi, E. Kanterakis an D. L. Schilling, "Multistage linear receivers for DS-CDMA systems," International Journal of Wireless Information Networks, Vol. 3, No. 1, pp.I-17, 1996.

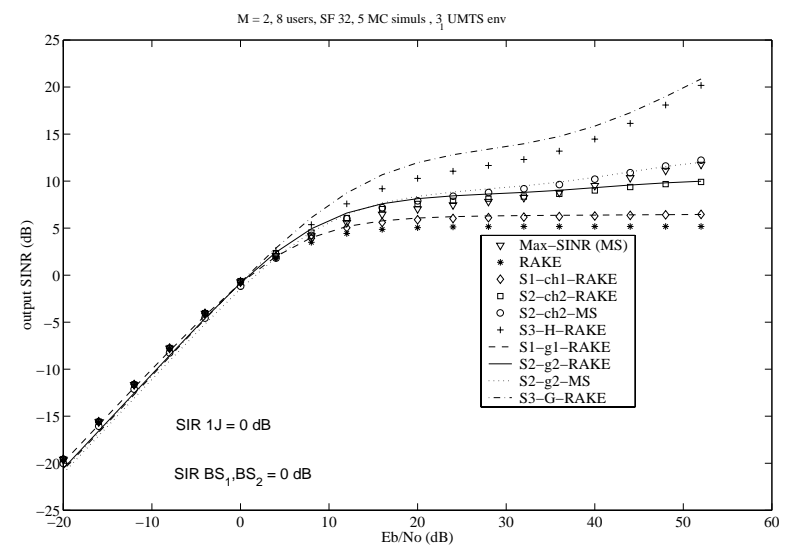

Figure 9. Output SINR versus Eb/No, 25\% loaded BSs, spreading factor 32 and equal power distribution, aperiodic scrambling, MMSE equalizers

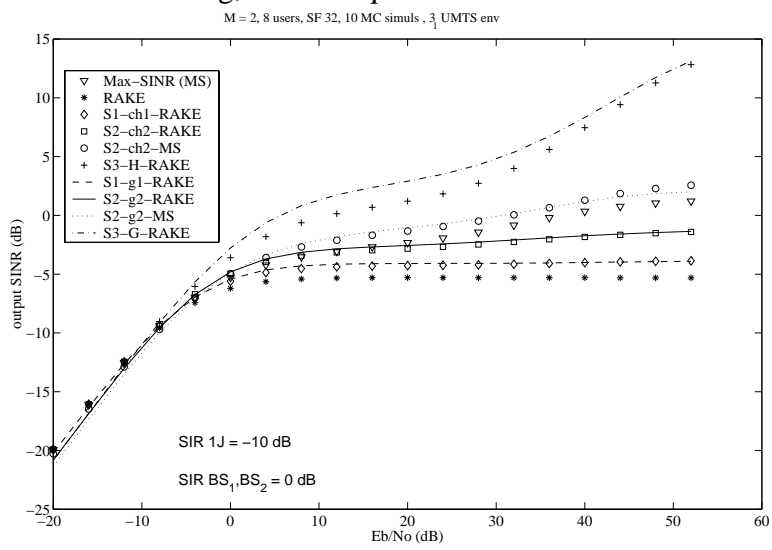

Figure 10. Output SINR versus Eb/No, 25\% loaded BSs, spreading factor 32 and near-far situation, aperiodic scrambling, MMSE equalizers

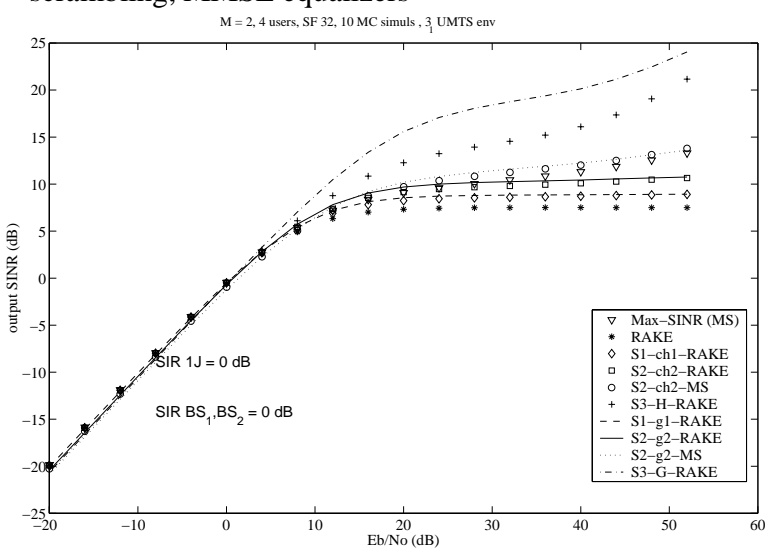

Figure 11. Output SINR versus Eb/No, $12.5 \%$ loaded BSs, spreading factor 32 and equal power distribution, aperiodic scrambling, MMSE equalizers

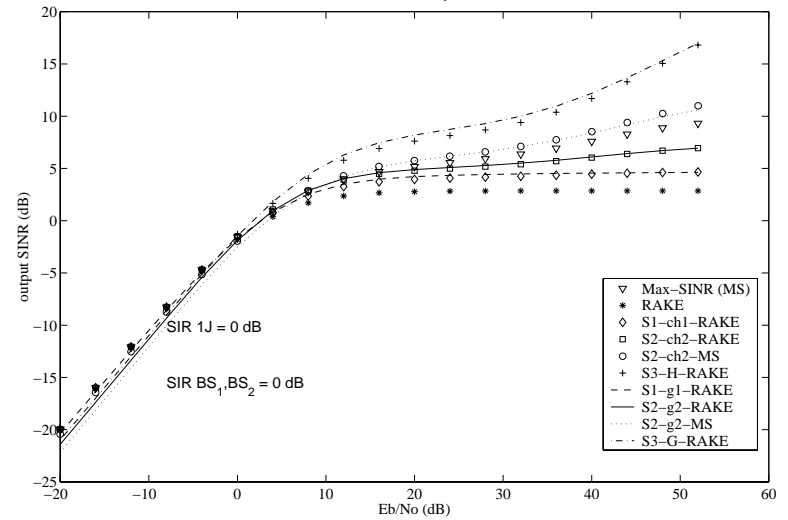

Figure 12. Output SINR versus Eb/No, $40.6 \%$ loaded BSs, spreading factor 32 and equal power distribution, aperiodic scrambling, MMSE equalizers

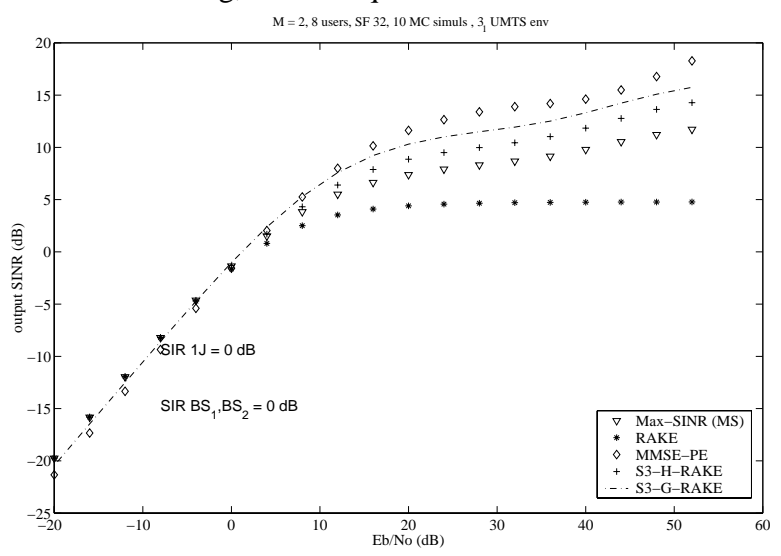

Figure 13. Output SINR versus Eb/No, 25\% loaded BSs, spreading factor 32 and equal power distribution, aperiodic scrambling, MMSE equalizers

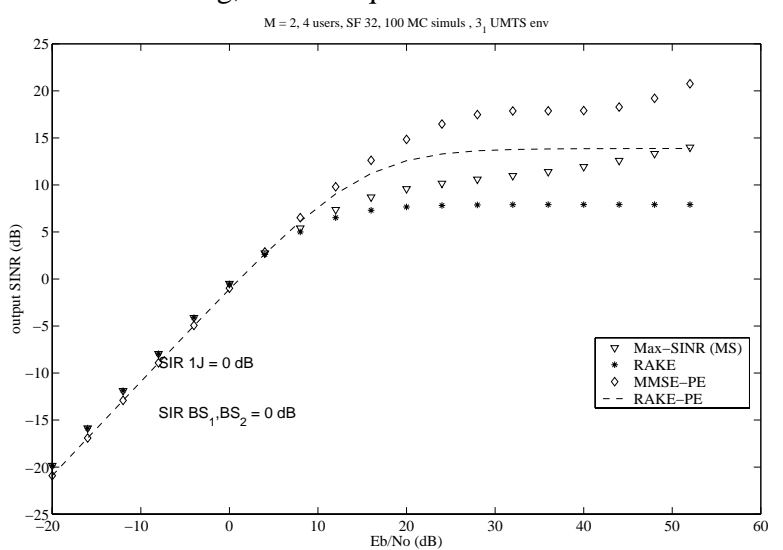

Figure 14. Output SINR versus Eb/No, $12.5 \%$ loaded BSs, spreading factor 32 and equal power distribution, aperiodic scrambling, MMSE equalizers

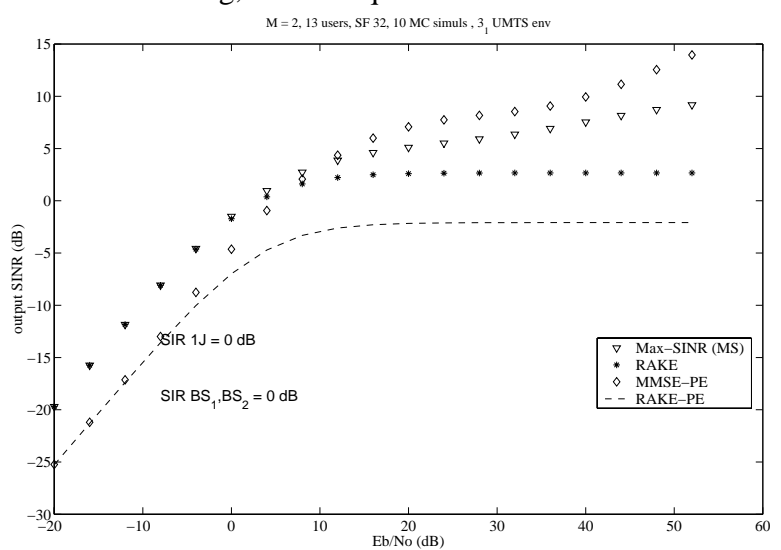

Figure 15. Output SINR versus Eb/No, $40.6 \%$ loaded BSs, spreading factor 32 and equal power distribution, aperiodic scrambling, MMSE equalizers 